https://helda.helsinki.fi

\title{
Estimating molar-incisor-hypomineralization among 8-year-olds based on 15-year public oral health practice-based data
}

\section{Tseveenjav, Battsetseg}

2020-10-02

Tseveenjav , B , Furuholm , J , Mulic , A, Valen , H, Maisala , T, Turunen , S, Varsio , S , Auero, $M$ \& Tjaderhane , L 2020 , ' Estimating molar-incisor-hypomineralization among 8-year-olds based on 15-year public oral health practice-based data ' , Acta Odontologica Scandinavica , vol. 78 , no. 7 , pp. 535-540 . https://doi.org/10.1080/00016357.2020.1751274

http://hdl.handle.net/10138/339906

https://doi.org/10.1080/00016357.2020.1751274

cc_by_nc

acceptedVersion

Downloaded from Helda, University of Helsinki institutional repository.

This is an electronic reprint of the original article.

This reprint may differ from the original in pagination and typographic detail.

Please cite the original version. 


\section{Estimating molar-incisor-hypomineralization among 8-year-olds based on 15-year public oral health practice-based data}

Authors and affiliations:

DDS, PhD Battsetseg Tseveenjav ${ }^{1,2,3}$; DDS Jussi Furuholm ${ }^{1,3}$, DDS, PhD Aida Mulic ${ }^{3}$; DDS, PhD Håkon Valen ${ }^{3}$; DDS Tuomo Maisala ${ }^{2}$, DDS Seppo Turunen², DDS, PhD Sinikka Varsio ${ }^{2}$, DDS Merja Auero ${ }^{4}$, Prof. Leo Tjäderhane ${ }^{1}$

${ }^{1}$ Department of Oral and Maxillofacial Diseases, University of Helsinki, Helsinki, Finland ${ }^{2}$ Oral Health Care, Department of Social Services and Health Care, City of Helsinki, Finland ${ }^{3}$ Nordic Institute of Dental Materials (NIOM), Oslo, Norway

${ }^{4}$ Ministry of Social Affairs and Health, Finland

Corresponding author:

DDS, PhD Battsetseg Tseveenjav,

P.O.B 41 (Mannerheimintie 172)

University of Helsinki, 00014, Helsinki, Finland

phone: +358503269119

e-mail: battsetseg.tseveenjav@helsinki.fi

Running title:

Estimating MIH

Word count:

3030 


\section{Abstract}

Background: A wide range in the prevalence of molar-incisor-hypomineralization (MIH) has been reported. Population-based studies are recommended. However, such studies are expensive and time-consuming.

Objectives: To estimate the magnitude of MIH condition among 8-year-olds based on routine oral health examinations and to associate first permanent molar (FPM) affection with that of other permanent teeth over time.

Materials and methods: This retrospective study, with cross-sectional and longitudinal components, was based on electronic oral health records; all 8-year-olds examined between 2002 and 2016 were included.

Results: The average estimated prevalence of MIH was $8.3 \%$; yearly range was $4.8 \%$ to $15.9 \%$. The mean number of affected teeth was $1.4 ; 62 \%$ had one affected tooth. One-surface defects were the most frequent (66\%). Asymmetric distribution of affected teeth was observed. In follow-up, 10.7\%, $8.4 \%$, and $11.2 \%$ had at least one affected permanent canine, premolar, or second permanent molar, respectively. The proportion of children with other $\mathrm{MIH}$-affected permanent teeth was higher in the group with $\geq 2 \mathrm{MIH}$-affected teeth than in group with one affected FPM at the age of 8 .

Conclusions: The average prevalence of MIH was comparable to that reported elsewhere. The number of MIH-affected teeth at early mixed dentition predicts the affection of other permanent teeth over time, mainly that of permanent canines. Further screening of children with MIH is recommended to improve individually tailored early preventive and restorative dental care.

Key words:

Prevalence, molar-incisor-hypomineralization, practice-based data, children, MIH affection of permanent canines 


\section{Introduction}

Molar-incisor-hypomineralization (MIH) is a dental developmental defect (DDD) of systemic origin of one or more first permanent molars (FPMs). MIH is frequently associated with a similarly affected permanent incisor(s) (PIs) [1]. To diagnose MIH, at least one FPM is required to be affected [2] regardless of involvement of PIs. MIH defects have also been observed in second primary molars, tips of permanent canine cusps, and second permanent molars [2-4].

MIH is a multifactorial condition due to a variety of systematically acting environmental factors, including prenatal, perinatal, and postnatal conditions [1, 5-11] with a possible underlying genetic predisposition [12]. Despite increasing knowledge, the aetiological evidence for MIH is insufficient to verify the proposed causative factors [13].

MIH-affected teeth present demarcated enamel opacities (DEOs) of different colours, from white to yellowish up to brown that occasionally undergo post-eruptive breakdowns [14]. DEOs are the most frequently encountered enamel defect among Swedish adolescents [15]; DEOs were $27 \%$ of the overall DDD prevalence (33\%), while the prevalence of diffuse opacities (5\%) and enamel hypoplasia (1\%) were irrelevant. Among Danish children of 6 to 8 years, nearly half had DEOs in FPMs or PIs [16].

A wide range in the prevalence of $\mathrm{MIH}(2.8 \%$ to $44 \%)$ has been reported. The estimated prevalence among different continents is $8.3 \%$ in Africa, $12.9 \%$ in Asia, $16.2 \%$ in Europe, $24.9 \%$ in Oceania, and 30\% in South America [17]. The wide range of prevalence rates may be due to differences in the age of the population, examination standards, chosen index, or calibration. In two recent meta-analyses, the average global prevalence of $\mathrm{MIH}$ was estimated at $13.1 \%$ [18] with significant differences between regions and countries and $14.2 \%$ [19] with no significant difference between boys and girls. Heavily populated countries contribute significantly to burden of prevalent cases, while growing ones rank first with respect to the number of incident cases [18]. 
In Nordic countries, the prevalence of MIH among Swedish cohorts of 11, 15, and 19 years was $17.1 \%, 11.2 \%$ and $8.3 \%$, respectively [15]. The prevalence in Norwegian adolescents of 16 years was $13.9 \%$ [3] and that of Finnish rural children (mean age 10 years) was $11.5 \%$ [20].

The Best Clinical Practice Guidance of the European Association of Paediatric Dentistry [2] recommends future population-based studies on the prevalence of MIH; the best group for a cross-sectional study are children of 8 years. However, population-based epidemiological studies are expensive and time-consuming, and therefore, the aim of the present study was to estimate for the first time the prevalence of MIH among children of 8 years that attended public oral health services in Helsinki based on 15-year cross-sectional practice-based data. In addition, MIH affection of other permanent teeth (other than FPM and PI) based on longitudinal follow-up of selected children was assessed over time.

\section{Material and methods}

This study was based on the large database of electronic health records of the Oral Health Care (OHC) of the City of Helsinki from 2002 to 2016. In Finland, as in other Nordic countries, oral health care is characterized by a large public sector that is subsidized by taxation and administered by municipalities. Thus, the $\mathrm{OHC}$ in Helsinki organizes the public oral health services for all inhabitants residing in the Helsinki area. All children and adolescents under 18 years are entitled to services without charge, including oral health examinations and treatments.

As the present study is a health register-based study, data were collected retrospectively from patient records of routine oral health examinations of children attending public health service. Data mining approach was used to collect the data of all children attending the city of Helsinki Public OHC service from the electronic patient health record system "EFFICA" (Tieto Oyj, Espoo, Finland). Encrypted summary data of all the visits, extracting all the variables included in the manuscript, was created and exported into Excel file and further into statistical software 
program file. Both practicing dentists and dental hygienists performed the oral health examinations and registered all clinical findings in the dental status chart, including caries and DDDs at the surface level of each tooth. Dental hygienists were required to consult dentists in case of doubt. Clinical examinations were carried out with well-equipped dental treatment unit with saliva suction and illumination, with examiners using a dental mirror and fibre optic light, while teeth surfaces being slightly wet. There was not any prior calibration of the examiners regarding registration of DDDs. However, examiners followed detailed guidelines to record findings of clinical oral examinations in the electronic patient records,.

For the cross-sectional component of this study, all children of 8 years undergoing oral health examinations between 2002 and 2016 were selected. Of this cohort, children with a MIH defect recorded in at least one FPM were eligible; the selected age was based on the recommendation of the Best Clinical Practice Guidance of EAPD [2]. Based on these criteria, 3042 children with MIH were selected. There were no children with 12 teeth affected (all FPMs and IPs at the same time), which thus excluded the possibility of major dental developmental conditions related to general health. The estimated prevalence of MIH was calculated for the present study subjects and weighted by gender proportion of the population of 8-year-old children residing in Helsinki (data obtained from Statistics Finland, www.tilastokeskus.fi/index_en.html).

MIH-affected teeth were described by tooth type, jaw location, size, and tooth surface location of the defects. The size of the defects was expressed by the number of tooth surfaces involved.

The inclusion criteria for the longitudinal component of this study were $\mathrm{MIH}$ at the age of 8 years and MIH affection of other permanent teeth other than FPMs and PIs in further oral health examinations. To be able to assess other permanent teeth other than FPMs and PIs, children of 8 years with MIH during the observation years 2002 to 2009 ( $n=2309)$ were eligible. Of these, within the framework of our data 1530 had another permanent tooth or other permanent teeth 
recorded as MIH affected in further oral health examination records between 2007 and 2016. Children in the follow-up group had a theoretical age range of 13 to 17 years. All teeth with MIH defects registered in examinations at the age of 13 to 17 years were cumulatively summed. In Finland, children are invited for oral health examinations by dentists or dental check-ups by dental hygienists based on age groups and caries risk and caries activity. Accordingly, not all children have an annual oral health examination. Therefore, to maximize the number of children selected for the follow-up component, an age range was given instead of exact age. The third molars were excluded if such teeth were registered.

\section{Statistical evaluation}

The extracted data were compiled into the statistical software package IBM SPSS Statistics for Windows (version 25.0, IBM Corp., Armonk, N.Y., USA) for statistical analyses. For descriptive statistics, $\chi^{2}$ test for differences of groups, t-test, and one-way ANOVA test for differences of means were used. A Cochran-Armitage test of trend was performed to assess the trend between year of examination and gender. A statistical level of 5\% was used for significance.

\section{Ethical considerations}

This study was based on encrypted summary data of electronic health records. The use of data for this study was approved by the City of Helsinki in Finland (Research permission decision registration number HEL-2017-000945). Individuals could not be identified, and ethical permission was therefore not applicable. Authors have no conflicts of interest.

\section{Results}

The average prevalence of MIH among children of 8 years in Helsinki was $8.3 \%$. The range was $4.8 \%$ to $15.9 \%$ based on a 15 -year observation period (Table 1 ). 
Of all children with MIH, $62 \%$ had one affected FPM, followed by $32 \%$ with two affected teeth. Table 2 shows the most common MIH-affected single tooth or teeth combinations among children of 8 years. Upper FPMs, namely D16 (35\%) or D26 (28\%), were the most frequently affected teeth among children with a single tooth affection. Among those with two affected teeth, the most frequent tooth combination was D16 with any other FPM (Table 2).

The mean number of MIH-affected teeth was $1.4(\mathrm{SD}=0.6$; range 1 to 5). The maxillary teeth were more often affected than the mandibular. An asymmetric distribution of MIHaffected teeth was observed; the right maxillary FPMs were 1.4 times more often affected than the left maxillary FPMs. The right maxillary central incisors were 2.6 times more commonly affected than the left maxillary central incisors. MIH-affected second primary molars were observed in 3.9\% of those with MIH condition. One-surface defects were the most frequent (66\%), followed by twosurface defects $(26.0 \%)$. The most common one-surface defects were located occlusally (36\%) or palatally (23\%) on D16, occlusally (34\%) and buccally (24\%) on D26, buccally (38\%) and occlusally (28\%) on D36, and occlusally (29\%) and buccally (28\%) on D46. The most common two-surface defects were located palato-occlusally on D16 and D26 (10\% in each) and buccoocclusally on D36 and D46 (18\% and 20\%, respectively).

Children at follow-up ( $\mathrm{n}=1530)$ had $3410 \mathrm{MIH}$-affected teeth (including FPMs and PIs); mean was $2.2(\mathrm{SD}=1.4)$. Figure 1 shows MIH-affected teeth of children at follow up as registered at the age of 8 years and in further oral health examinations.

Of all children, $10.7 \%$ had at least one permanent canine affected, $8.4 \%$ had at least one premolar affected, and $11.2 \%$ had at least one second permanent molar affected. The proportion of children with an MIH-affected permanent canine, premolar, or second permanent molar at follow-up was higher among those who had two or more MIH-affected teeth than those with only one FPM affected at the age of 8 years (Figure 2). 


\section{Discussion}

This study estimated for the first time the prevalence of MIH in the Helsinki area based on population-based electronic health record data. The estimated average prevalence $(8.4 \%)$ of $\mathrm{MIH}$ among children of 8 years in Helsinki based on the 15-year period is consistent with the prevalence reported for children of mean age of 10 years in two rural Finnish towns [20] and Spanish schoolchildren of 6 to 14 years [9]. However, the observed prevalence was lower than that reported in other Northern European countries, such as Norway [3], Sweden, [15] and Denmark [16]. However, the children's age groups in these studies differed.

The main finding of the longitudinal part of this study was that the number of MIHaffected teeth at the age of 8 years predicted to the affection of other permanent teeth over time. This knowledge needs to be taken into consideration in tailoring individual dental check-up intervals for children.

Estimation of MIH in Helsinki was far lower than reported prevalence in regions and countries with heavily crowded populations, such as India, China and the United States [18]. Burden of MIH in these countries might be partly explained by socio-environmental differences, such as diet and pollution as well as access to health care.

In the present study, the typical asymmetric feature of MIH affection in the dentition was seen. The maxillary FPMs were 1.4 times more often affected than the mandibular FPMs, consistent with the frequency reported (1.6 times) among Norwegian children [3]. The proportion of children with a single tooth or molar affection among MIH children was much higher in our study $(62 \%)$ than that reported for Norwegian adolescents of 16 years $(31.8 \%)$ [3].

No gender difference of MIH was observed in our study population. This is consistent with the two reviews, which included 99 [18] and 70 studies [19], respectively, though the former one highlighted observation of higher prevalence in girls than boys in South Asia. Similarly, 
prevalent cases in girls were also reported among Norwegian adolescents of 16 years [3], and Spanish schoolchildren of 6 to 14 years [9].

In the present study, only few children had a MIH-affected second primary molar. This is perhaps an underestimation of possible affection of second primary molars, as at this age most primary molars with developmental defects might be restored or extracted. An optimal age of 5 years is recommended for diagnosis of developmental defects in primary molars.

The strength of this study is that the data were obtained from a large population-based database of public service, which practically all children under 18 years attend. According to Statistics Finland, during the years 2002 to 2016 the yearly cohort of children of 8 years residing in Helsinki ranged from 4820 to 6080 children, with a total cumulative sum of 78204 children (www.tilastokeskus.fi/index_en.html); of these, 36753 children were undergoing oral health examinations in the public health care setting. Thus, the prevalence of MIH was based on $47 \%$ of the population of 8 -year-olds over the 15 -year observation period. Therefore, this is a representative estimate of the prevalence of MIH and the magnitude of MIH-affected teeth. Our study population was far larger than the EAPD recommendation for epidemiological studies of MIH, which should be at least 300 people [2]. Another strength of this study is the design. Based on 15-year populationbased data, the trend of MIH for the entire observation period was explored. The longitudinal follow-up component allowed assessment for the first time the association of MIH-affected teeth of the early mixed dentition period with those of permanent dentition in the same children.

The estimated average prevalence of the present study may be an underestimation as not all defects are necessarily registered due to possible unawareness among dental professionals regarding MIH in general, diagnostic dilemmas the examiner may encounter during oral health examinations, or both. In turn, yearly differences seen in prevalence might be explained partly by variation of clinicians' knowledge and awareness. 
On the other hand, the determined prevalence may be an overestimation as other developmental defects such as diffuse opacities and enamel hypoplasia might have been diagnosed as MIH defects, as the former may be confused with the latter with post-eruptive breakdown or onset of caries. However, demarcated opacities are the most common DDDs among children and adolescents $[15,16]$. It is worth mentioning that even in clinical studies of DDDs, differentiation of tooth tissue loss due to post-eruptive breakdown of demarcated opacities and enamel hypoplasia (which is a quantitative defect of enamel) may be questionable [15].

The limitation of this study is that any misdiagnosis or failure to record MIH defects was handled as they were in patient records, as any other patient record-based study. Therefore, health record-based studies are usually not as precise as epidemiological studies based on clinical examinations. However, the expenses for data collection are far lower and as mentioned above the power of patient-register studies is in high number of examined subjects.

It is worth mentioning also that counting MIH-affected teeth over time was not straightforward. Some new FPMs and especially new PIs with MIH defects were registered at follow up, possibly because of better visibility of all tooth surfaces at later age due to either full eruption of those teeth or improved oral hygiene. This may partly explain the discrepancies in number of MIH teeth at the age of 8 years and in follow-up.

As caries rates have declined in Western countries during the last decades, DDDs among children are becoming more apparent and require more complex and long-term treatment options [1]. Comparing the prevalence of MIH across countries is a challenging task as the study subjects selected were not homogeneous in age. When assessing the prevalence of MIH at the population level, the age groups need to be well-defined for the purpose. 


\section{Conclusions}

The average prevalence of MIH among children of 8 years in Helsinki was comparable to that reported elsewhere. An irrelevant number of children had MIH-affected second primary molars. The number of MIH-affected teeth at early mixed dentition predicts the affection of other permanent teeth other than FPM and PI over time. Further screening of children diagnosed with MIH at the age of early mixed dentition would improve targeting of individually tailored preventive measures. This will facilitate management of further clinical dental problems of affected other permanent teeth and improve oral health-related quality of life of children with this condition.

\section{Acknowledgements}

We would like to thank Professor Jon E Dahl, managing director of NIOM for valuable comments and support during this project. Author BT thanks the Finnish Dental Society for research grant.

\section{Declaration of interest}

The authors report no conflicts of interest. The authors alone are responsible for the content and writing of the paper. 


\section{References}

Lygidakis NA. Treatment modalities in children with teeth affected by molar-incisor enamel hypomineralisation (MIH): A systematic review. Eur Arch Paediatr Dent 2010;11(2):65-74.

Lygidakis NA, Wong F, Jalevik B, et al. Best Clinical Practice Guidance for clinicians dealing with children presenting with Molar-Incisor-Hypomineralisation (MIH): An EAPD Policy Document. Eur Arch Paediatr Dent 2010;11(2):75-81.

Schmalfuss A, Stenhagen KR, Tveit AB, et al. Canines are affected in 16-year-olds with molar-incisor hypomineralisation (MIH): an epidemiological study based on the Tromso study: "Fit Futures". Eur Arch Paediatr Dent 2016;17(2):107-13.

Garot E, Denis A, Delbos Y, et al. Are hypomineralised lesions on second primary molars (HSPM) a predictive sign of molar incisor hypomineralisation (MIH)? A systematic review and a meta-analysis. J Dent 2018;72:8-13.

Silva MJ, Scurrah KJ, Craig JM, et al. Etiology of molar incisor hypomineralization A systematic review. Community Dent Oral Epidemiol 2016;44(4):342-53.

Willmott NS, Bryan RA, Duggal MS. Molar-incisor-hypomineralisation: a literature review. Eur Arch Paediatr Dent 2008;9(4):172-9.

Wogelius P, Haubek D, Nechifor A, et al. Association between use of asthma drugs and prevalence of demarcated opacities in permanent first molars in 6-to-8-year-old Danish children. Community Dent Oral Epidemiol 2010;38(2):145-51.

Wu X, Wang J, Li YH, et al. Association of molar incisor hypomineralization with premature birth or low birth weight: systematic review and meta-analysis. J Matern Fetal Neonatal Med 2018:1-9.

Hernandez M, Boj J, Espasa E, et al. Molar-Incisor Hypomineralization: Positive Correlation with Atopic Dermatitis and Food Allergies. J Clin Pediatr Dent 2018;42(5):344-8. 
status and enamel hypomineralization. Community Dent Oral Epidemiol 2018;46(4):343-51.

Kuhnisch J, Mach D, Thiering E, et al. Respiratory diseases are associated with molarincisor hypomineralizations. Swiss Dent J 2014;124(3):286-93.

Vieira AR, Kup E. On the Etiology of Molar-Incisor Hypomineralization. Caries Res 2016;50(2):166-9.

Alaluusua S. Aetiology of Molar-Incisor Hypomineralisation: A systematic review.

Eur Arch Paediatr Dent 2010;11(2):53-8. Weerheijm KL. Molar incisor hypomineralisation (MIH). Eur J Paediatr Dent 2003;4(3):114-20. Jalevik B, Szigyarto-Matei A, Robertson A. The prevalence of developmental defects of enamel, a prospective cohort study of adolescents in Western Sweden: a Barn I TAnadvarden (BITA, children in dental care) study. Eur Arch Paediatr Dent 2018.

Wogelius P, Haubek D, Poulsen S. Prevalence and distribution of demarcated opacities in permanent 1st molars and incisors in 6 to 8 -year-old Danish children. Acta Odontol Scand 2008;66(1):58-64.

Hernandez M, Boj JR, Espasa E. Do We Really Know the Prevalence of MIH? J Clin Pediatr Dent 2016;40(4):259-63. Schwendicke F, Elhennawy K, Reda S, et al. Global burden of molar incisor hypomineralization. J Dent 2018;68:10-8. Dave M, Taylor G. Global prevalence of molar incisor hypomineralisation. Evid Based Dent 2018;19(3):78-9. Wuollet E, Laisi S, Salmela E, et al. Molar-incisor hypomineralization and the association with childhood illnesses and antibiotics in a group of Finnish children. Acta Odontol Scand 2016;74(5):416-22. 


\section{Tables}

Table 1: Estimated prevalence of molar-incisor-hypomineralization (MIH) among children of 8 years $(n=3042)$ in Helsinki during 2002-2016

\begin{tabular}{|c|c|c|c|c|c|c|c|}
\hline \multirow[b]{2}{*}{ year } & \multicolumn{2}{|c|}{$\begin{array}{c}\text { Children of } 8 \text { years } \\
\text { with MIH }\end{array}$} & \multicolumn{3}{|c|}{$\begin{array}{c}\text { Estimated prevalence of } \\
\text { MIH } \\
\text { in present study } \\
\text { population } \\
\%\end{array}$} & \multicolumn{2}{|c|}{$\begin{array}{c}\text { Weighted estimated } \\
\text { prevalence } \\
\text { of MIH } \\
\%\end{array}$} \\
\hline & $\underset{\mathrm{n}}{\mathrm{MIH}}$ & $\begin{array}{c}\text { Boys } \\
\mathrm{n}\end{array}$ & All & Boys & Girls & Boys & Girls \\
\hline 2002 & 400 & 203 & 15.9 & 15.7 & 16.0 & 15.9 & 15.9 \\
\hline 2003 & 267 & 140 & 9.1 & 9.6 & 8.7 & 9.3 & 8.9 \\
\hline 2004 & 155 & 93 & 6.3 & 7.3 & 5.3 & 7.2 & 5.4 \\
\hline 2005 & 288 & 161 & 11.6 & 13.3 & 10.0 & 13.1 & 10.1 \\
\hline 2006 & 238 & 132 & 10.8 & 11.7 & 9.9 & 11.7 & 9.9 \\
\hline 2007 & 246 & 123 & 10.6 & 10.6 & 10.6 & 10.5 & 10.7 \\
\hline 2008 & 189 & 99 & 6.3 & 6.5 & 6.0 & 6.5 & 6.0 \\
\hline 2009 & 152 & 72 & 5.9 & 5.5 & 6.4 & 5.5 & 6.4 \\
\hline 2010 & 188 & 100 & 7.3 & 7.5 & 7.1 & 7.4 & 7.2 \\
\hline 2011 & 186 & 98 & 6.9 & 7.2 & 6.6 & 7.2 & 6.5 \\
\hline 2012 & 125 & 57 & 4.8 & 4.4 & 5.2 & 4.3 & 5.4 \\
\hline 2013 & 132 & 67 & 5.3 & 5.4 & 5.2 & 5.3 & 5.3 \\
\hline 2014 & 167 & 92 & 7.5 & 8.1 & 6.8 & 8.1 & 6.9 \\
\hline 2015 & 135 & 82 & 6.1 & 7.0 & 5.2 & 7.2 & 5.0 \\
\hline 2016 & 174 & 85 & 12.0 & 11.6 & 12.5 & 11.6 & 12.5 \\
\hline All & 3042 & 1604 & 8.3 & 8.6 & 8.0 & 8.7 & 8.3 \\
\hline
\end{tabular}


Table 2: Most common MIH-affected tooth or teeth combinations among 8-year-olds $(n=3042)$

\begin{tabular}{|c|c|c|c|}
\hline & $\begin{array}{l}\text { Most common tooth or teeth } \\
\text { combinations }\end{array}$ & $\mathrm{N}$ & $\begin{array}{c}\% \\
\text { within } \\
\text { each group } \\
\end{array}$ \\
\hline $\begin{array}{l}\text { One tooth affected } \\
\mathrm{n}=1894 \\
(62 \%)\end{array}$ & $\begin{array}{l}\text { D16 } \\
\text { D26 } \\
\text { D36 } \\
\text { D46 }\end{array}$ & $\begin{array}{l}658 \\
536 \\
379 \\
321\end{array}$ & $\begin{array}{l}35 \% \\
28 \% \\
20 \% \\
17 \%\end{array}$ \\
\hline $\begin{array}{l}\text { Two teeth affected } \\
\mathrm{n}=957 \\
(32 \%)\end{array}$ & $\begin{array}{l}\text { D16 and D26 } \\
\text { D16 and D46 } \\
\text { D16 and D36 } \\
\text { D26 and D46 } \\
\text { D26 and D36 } \\
\text { D36 and D46 } \\
\text { D16 and D11 } \\
\text { D36 and D11 } \\
\text { D26 and D11 } \\
\text { D46 and D11 } \\
\text { Other combinations } \\
\text { (each combination had }<21 \text { cases) }\end{array}$ & $\begin{array}{c}149 \\
116 \\
90 \\
67 \\
56 \\
48 \\
42 \\
30 \\
30 \\
21 \\
308\end{array}$ & $\begin{array}{l}16 \% \\
12 \% \\
9 \% \\
7 \% \\
6 \% \\
5 \% \\
5 \% \\
3 \% \\
3 \% \\
2 \% \\
32 \%\end{array}$ \\
\hline $\begin{array}{l}\text { Three teeth affected } \\
\mathrm{n}=176 \\
(6 \%)\end{array}$ & $\begin{array}{l}\text { D16, D26, and D46 } \\
\text { D16, D26, and D36 } \\
\text { D16, D36, and D46 } \\
\text { D16, D46, and D11 } \\
\text { D16, D36, and D11 } \\
\text { Other combinations } \\
\text { (each combination had <5 cases) }\end{array}$ & $\begin{array}{c}27 \\
9 \\
9 \\
7 \\
6 \\
118\end{array}$ & $\begin{array}{c}14 \% \\
5 \% \\
5 \% \\
3 \% \\
3 \% \\
70 \%\end{array}$ \\
\hline $\begin{array}{l}\text { Four teeth affected } \\
\mathrm{n}=14 \\
(<1 \%)\end{array}$ & $\begin{array}{l}\text { No common combination } \\
\text { (14 different combinations) }\end{array}$ & 11 & $100.0 \%$ \\
\hline $\begin{array}{l}\text { Five teeth affected } \\
\mathrm{n}=1 \\
(<0.1 \%)\end{array}$ & Combination: D22, D26, D41, D42, D46 & 1 & $100.0 \%$ \\
\hline All & & 3042 & \\
\hline
\end{tabular}


Figure 1: MIH-affected teeth at the age of 8 years and in other permanent teeth over time among children at follow-up $(\mathrm{n}=1530)$.
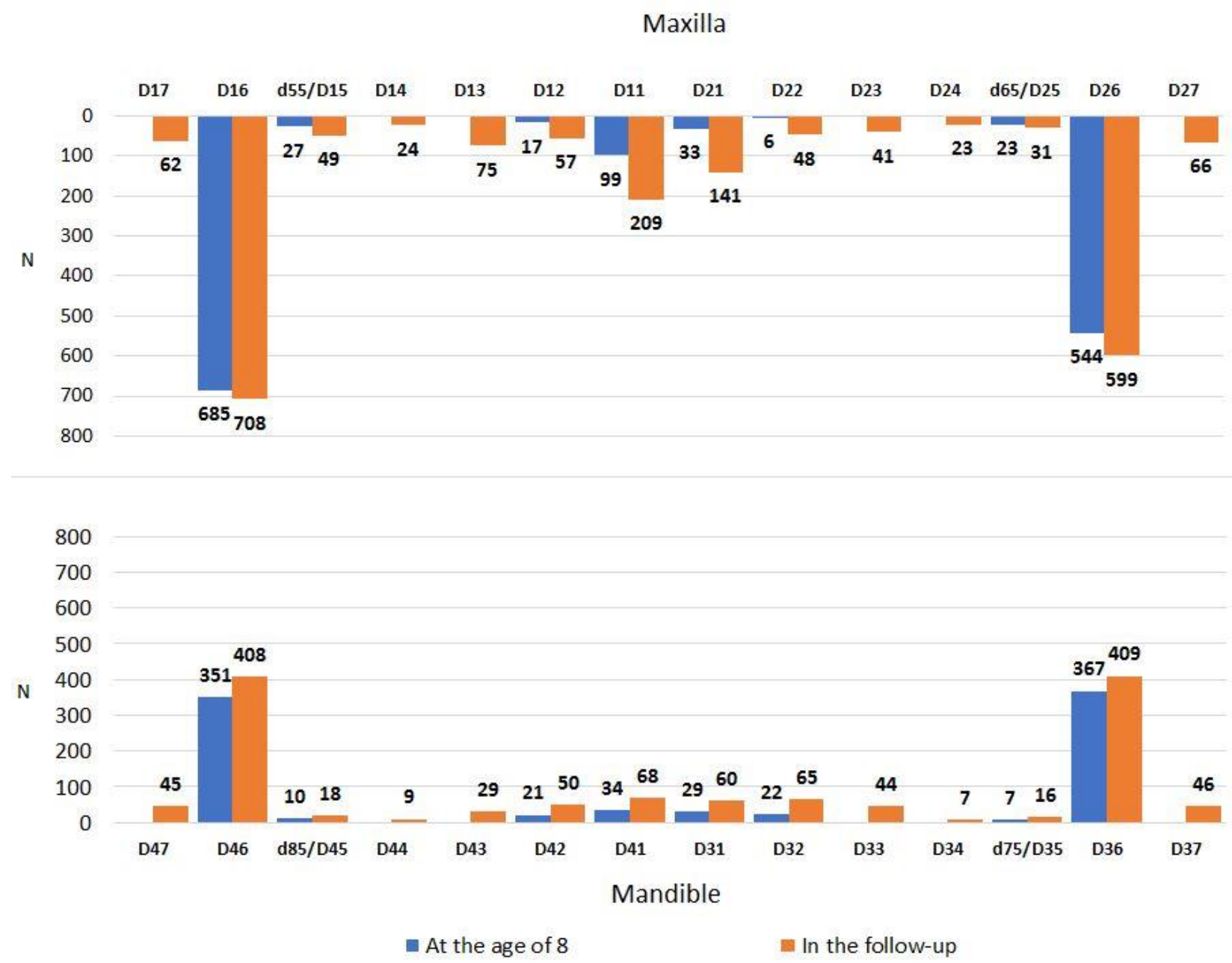

Figure 2: Proportion (\%) of children with MIH-affected other permanent teeth at follow-up. 
Proportion of children with $\mathrm{MIH}$ affected other permanent teeth over time according to their affected teeth at the age of 8 years

$25 \%$

$20 \%$

$15 \%$

$10 \%$

$5 \%$

$0 \%$
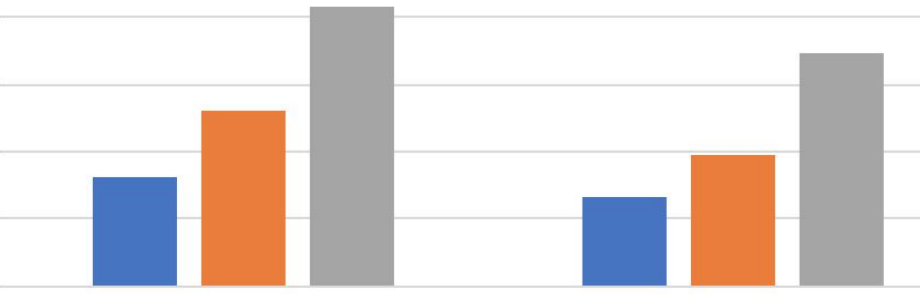

At least one permanent canine affected over time

At least one premolar affected over time
At least one second permanent molar affected over time

In the follow-up

One tooth affected at the age of 8

Two teeth affected at the age of 8

Three or more teeth affected at the age of 8 